\title{
22
}

\section{Interactive simulation for planning, coordination and control: Mathematical decision support systems in education}

\author{
B. E. Wholeben
}

\author{
College of Education, Northern Illinois University, DeKalb Illinois 60115 USA
}

Complexities in educational management, inherent in the strategic planning of multiple alternatives as well as the tactical control of limited resources, encourage a continued restructuring and export of industrial engineering methods for the educational domain. Such engineering methods, defined broadly as operations research or mathematical modeling techniques, provide a valuable diversity of technical approaches to organizing, analyzing, and interpreting the vast amounts of data stored in electronic management information systems. Given the availability of technology in the forms of high-speed personal computers and lowcost decision modeling software, competing alternative decisions may readily be simulated in order to measure each alternative's impact upon the operating policy in an educational governance system.

Keyword Codes: H.2.8; I.6.7; K.3.1

Keywords: Database Applications; Simulation Support Systems; Computer Uses in Education

\section{INTRODUCTION}

The management of educational practice and process is an inherently complex and complicated task in today's post-modern societies. From problem identification to solution validation, an increasingly scientific approach to problem solving in today's educational sector is necessary to satisfy constituency demands, that the wide range of available data be incorporated into each decision. The wide spread development of management information systems which plan, coordinate, and control daily educational practice and process offers to the educational manager an opportunity to incorporate available data into tactical and strategic decision making.

\subsection{Decision support systems}

When the available data in an organized management information system is used in a systematic way to assist decision making, that management information system may be referred to as a decision support system. Basic management information systems collect routine data which can be used to monitor operations, and thereby suggest to the manager, when operations deviate from acceptable practice, that some modification to typical practice (decision) is necessary. It is the manager's interpretation of the deviation recorded by the management information system which suggests to the manager a solution (decision) to reverse the effects of a problem (deviation). A decision support system is an extension of the management information system, in that the data collected by a decision support system not 
only alert managers to deviations from acceptable practice, but in addition, construct feasible alternative solutions which the manager may reflect upon before making a final decision (choice from among these feasible alternative solutions).

\subsection{Mathematical modeling}

In order to construct feasible alternative solutions, the decision support system reconstructs the "problem setting" as an interrelationship between data in the management information system portion of the decision support system and the collectively exhaustive components of competing alternative solutions. The interrelationship between data and solutions, usually expressed mathematically in the form of a cross-reference matrix, constructs a model which can trace the interaction between data representing the problem and data defining the solution. This interaction provides the basis for a decision support system to compare alternative solutions for their strengths and limitations in meeting the needs of an identified problem. "Best" solutions (viz., optimal solutions) are then suggested to the manager by the decision support system. Suggested solutions are based upon each competing solution's effectiveness in meeting pre-established guidelines or criteria. By simulating the consequences of each potential solution, given this competitive interaction between (among) data-based alternatives, the degree of each alternative's effectiveness as a solution may be measured.

\subsection{Interactive simulation}

Interactive simulation, the ability to test the suitability of alternative solutions given what is known about the problem setting, is a formidable component of a mathematical model constructed in the decision support system. As conditions change, the data measured by the management information system portion of the decision support system also change, and the influence of each alternative solution profiled by such data likewise changes.

\section{MODELS FOR EDUCATIONAL MANAGEMENT}

This paper explores, and gives examples of, mathematically formulated, interactive simulation models which can be constructed as part of a decision support system for educational management. These interactive simulation models identify a class of problems in which a probable solution is defined as a finite, mutually exclusive group of "subsolutions" (or partial solutions) which comprise collectively the optimal solution to a problem. Three approaches to subsolution modeling are examined in this paper: periodic probability transitions, hierarchical linear clusters, and precedent activity mapping.

\subsection{Periodic probabilistic transition model}

The periodic probabilistic transition model structures the problem setting as predictable stage-to-stage transformations of objects within a standardized time period. These prediction models provide on-demand the ability to assess the effectiveness of on-going programs which have random-access entry phases of subject (object) entry. Examples of problems in which probabilistic transition modeling has promoted utilitarian solutions follow.

- Determine the predictable impact of a multi-faceted behavioral discipline program on subsequent behavior and categorical recidivism rates.

- Determine the predictable expectancy of course registrations for the development of multi-year course offering schedules based upon demand precedence history. 
- Determine the predictable balloting habits of school board members in upcoming policy referenda based upon past consensus decisions by issue of consensual question.

\subsubsection{Technique}

A square matrix is constructed wherein rows represent the 'from-states' which subjects (objects) depart, and columns the 'to-states' which subjects enter. Matrix cells contain the conditional probabilities of these "from-to" periodic transitions. Therefore, each row must sum to 1.0 , since all transition possibilities must be accounted for. If any transition places a subject into a state from which the subject can not depart to any of the other states (absorbed), the model is complicated and requires additional interpretation as compared to a non-absorbing model. The initial matrix contains observed conditional probabilities which represent the historical transitions between state 1 and state 2, thus comprising period 1 . Next, the matrix is raised exponentially to a power which represents the targeted period under study. For example, if the effect of the 1 st transition is of interest, the matrix is raised to the power of 2 (squared), and the resulting cell values are interpreted as expected conditional probabilities for each transition between states in period 2. That is, raising the matrix through matrix multiplication to a power of $\{x\}$ results in the calculation of conditional probabilities expected to exist as a result of period $\{x\}$. Repeated exponentiation of the initial conditional probability matrix will eventually result in a matrix wherein the values of a particular column (to-state) will be identical. When this occurs, the model is said to have reached equilibrium, or be in its steady state. The value of the exponent required to achieve model equilibrium, and the values of the steady-state probabilities which result, are significant for interpreting the underlying concept (construct) modeled by the periodic probabilistic transition model. Simulation is achieved by altering the conditional probabilities associated with the initial transition matrix based upon suggested changes in policy; or, alternatively, by adding new transition states to the model in order to evaluate potential impact. Decision support status is achieved when the steady-state values satisfy existing policy, or these same values are compared with the equilibrium of a competing periodic probabilistic transition model in order to determine optimal period probabilities.

\subsubsection{Example}

A vocational trades school which serves a large urban school district as a specialty (magnet) school -- enrolling only high school students who have been referred from other high schools because of continuing behavior problems -- has for one academic 10-week term piloted a highly structured, behavioral modification program in terms of graduated levels of punishment based upon repeated or severity of the cited offense. This discipline program has five levels, ranging from a verbal reprimand to expulsion. Students who are expelled are required to re-enroll the subsequent semester. Students who are not presently under any disciplinary censure are considered in-good-standing. A student's disciplinary rating is assessed as the highest disciplinary action adjudicated during the 10-week period. At the conclusion of this 10-week pilot, conditional probabilities were computed, and entered into a periodic probabilistic transition model as shown in Figure 1 .

At a power of 10 the model illustrated in Figure $l$ reached equilibrium. Therefore, all things remaining equal, the conditional probabilities computed from the 10 -week program pilot suggest that, by the conclusion of the 10th 10 -week use of this graduated behavior discipline program, the steady-state probabilities for each state in this discipline program may be expected as those values displayed in Figure 2. 
Figure 1. Conditional probabilities for student transitions resulting from 10-week pilot.

\begin{tabular}{|c|c|c|c|c|c|c|c|}
\hline Discipline Program Options & IGS & VER & DET & STS & LTS & EXP & RET \\
\hline IGS IN-GOOD-STANDING & .36 & .25 & .05 & .10 & .15 & .09 & -- \\
\hline VER VERBAL REPRIMAND & .44 & .15 & .10 & .20 & .07 & .04 & -- \\
\hline DET DETENTION & .50 & .10 & .05 & .05 & .15 & .15 & -- \\
\hline STS SHORT-TERM SUSPENSION & .65 & .05 & .05 & .01 & .20 & .04 & -- \\
\hline LTS LONG-TERM SUSPENSION & .15 & .25 & .15 & .05 & .10 & .30 & -- \\
\hline EXP EXPULSION & -- & -- & -. & -. & -- & -- & 1.0 \\
\hline RET REINSTATEMENT & .7 & .05 & .05 & .05 & .05 & .10 & -- \\
\hline & & $\diamond$ & & & & & \\
\hline Discipline Program Options & IGS & VER & DET & STS & LTS & EXP & RET \\
\hline EQUILIBRIUM & .38 & .16 & .06 & .09 & .11 & .10 & .10 \\
\hline
\end{tabular}

Therefore, once model equilibrium is achieved, $38 \%$ of all enrolled students can be expected to be in-good-standing during any given period, while $16 \%$ of all enrolled students can be expected to have received a verbal reprimand as their highest level of disciplinary censure during any given period. These steady-state values can be utilized for benchmarking purposes. Should any changes to the entry-exit criteria occur, which define each of the graduated disciplinary censure states, recomputation of the model will produce a revised set of steady-state values for comparison with the pilot (benchmark) values.

\subsection{Hierarchical linear cluster model}

The hierarchical linear cluster model structures the problem setting as groups (clusters) of competing (hierarchical) elements. Hierarchical clusters provide an ability to evaluate the best-mix of potential decision (solution) alternatives which (a) satisfy the valued constraints in a given decisioning situation, and (b) optimize (maximize or minimize) some particularly important dependent (positive or negative) objective. Examples of problems in which hierarchical modeling has promoted utilitarian solutions follow.

- Determine which funded components in each program budget (hierarchical cluster) should be increased or decreased, and by how much, given strategic education missior documented needs, and overall available resources.

- Determine which geocoded residential addresses of students should be assigned to which school campus, given an enrollment catchment plan (hierarchical cluster) which must balance enrollments with building capacity, minimize student transportation time, and satisfy affirmative action requirements associated with the socio-economic status and ethnic diversity of student families.

- Determine the best hardware and software combinations (hierarchical cluster) to support computer-assisted instruction, given desired student learning activities by instructional objective across interdisciplinary curricula. 


\subsubsection{Technique}

A system of simultaneous linear equations defines a tableau with a rectangular constraint matrix including appended bounded limits (column vector), and an objective function (row vector) to optimize the evaluation of feasible alternative solutions. The multiple equalities and inequalities (rows) in the constraint matrix provide the basis for modeling the criteria for decision making as defined by policy. Bounded limits for each equation or inequality specify the goals of the particular policy modeled. Coefficients in the constraint matrix supply the competitive values of various decision options for modeling as defined by the policy constraints. The constraint matrix identifies which combinations of alternative decisions are feasible (operational) within policy limitations; then, the objective function (vector) selects the "best" alternative by maximizing (minimizing) some a priori identified positive (negative) variable according to the stated mission of the hierarchical linear cluster model. Decision variables are dependent variables, and serve to identify the various options (alternatives) which are available to be incorporated into the final solution, and will be assigned a value of either $\{1\}$ or $\{0\}$ as a result of model calculations. $\{1\}$ means that this option will be included as part of the final solution; $\{0\}$ that the option not be a part of the final solution. A second class of dependent variables, accumulator variables, serve to store total benefits and limitations of the various 'mix' of decision options for re-input to the management information system for reporting purposes. Simulation is achieved by altering the bounded limits associated with the constraint matrix, based upon exploratory discussions of the human decision making; or alternatively, by adding new options for considering in the mix of already available options. Decision support system status is achieved when the model outputs the specific components of an optimal solution.

\subsubsection{Example}

A state education budget oversight committee is charged with the responsibility for suggesting budgetary revisions to present state contributions to local education agency budgets. As a pilot, two LEAs with significantly contrasting budgetary elements have been identified to test the use of a hierarchical linear cluster approach to solution development. The solution, in this case, is to identify what reallocations should be recommended relative to each of these two operating budgets. A partial model is presented in Figure 3 for purposes of illustration.

This illustration depicts the two program budgets [1.1] with their respective options for budgetary revision [1.2]. The accumulator variables $\{\Sigma X, \Sigma Y, \Sigma Z\}$ on [1.1] specify re-entry to the issuing management information system. One and only one budgetary option can be selected for each budget [2.1-2.2], and the amount of planned budgetary expenditures must not exceed total monies available [3.1]. The extent to which a mix of feasible alternatives' compliance with stated policy goals is returned to the management information system for reporting purposes [4.1-4.3], depends upon the determination of the appropriateness of each mix of feasible alternatives as a probable solution [5.1-5.3]. The final selection of the optimal budgetary solution, the best mix of alternative budgetary options, is made by maximizing (minimizing) the total value of a particular variable [6.1].

\subsection{Precedent activity mapping model}

The precedent activity mapping model structures the problem setting as an exhaustive collection of tasks which must be accomplished to complete an objective. A solution is defined by that finite number of linked (predecessor-to-successor) activities (tasks) which 


\section{Figure 3. Sample tableau formulation of the hierarchical linear cluster model.}

\begin{tabular}{|c|c|c|c|c|c|c|c|c|c|}
\hline 1.1 & - & $\cdots A$ & -..-- & ----- & 3 -..-- & $\Sigma \mathrm{X}$ & $\Sigma Y$ & $\Sigma Z$ & \\
\hline 1.2 & 1 & 2 & 3 & 1 & 2 & & & & \\
\hline 2.1 & 1 & 1 & 1 & & & & & & $=1$ \\
\hline 2.2 & & & & 1 & 1 & & & & $=1$ \\
\hline 3.1 & $\$ \$ \$_{A 1}$ & $\$ \$ \$_{\mathrm{A} 2}$ & $\$ \$ \$_{\mathrm{A} 3}$ & $\$ \$ \$_{B 1}$ & $\$ \$ \$_{B 2}$ & & & & $\leq \$ \$ \$_{\mathrm{TOT}}$ \\
\hline 4.1 & $\mathrm{X}_{\mathrm{A} 1}$ & $\mathrm{X}_{\mathrm{A} 2}$ & $\mathrm{X}_{\mathrm{A} 3}$ & $X_{131}$ & $X_{132}$ & -1 & & & $=0$ \\
\hline 4.2 & $\mathrm{Y}_{\mathrm{A} 1}$ & $\mathrm{Y}_{\mathrm{A} 2}$ & $Y_{A 3}$ & $Y_{B 1}$ & $Y_{B 2}$ & & -1 & & $=0$ \\
\hline 4.3 & $\mathrm{Z}_{\mathrm{A} 1}$ & $\mathrm{Z}_{\mathrm{A} 2}$ & $\mathrm{Z}_{\mathrm{A} 3}$ & $\mathrm{Z}_{131}$ & $\mathrm{Z}_{\mathrm{B} 2}$ & & & -1 & $=0$ \\
\hline 5.1 & & & & & & 1 & & & $\geq$ GOAL $X$ \\
\hline 5.2 & & & & & & & 1 & & $\geq$ GOAL Y \\
\hline 5.3 & & & & & & & & 1 & $\geq \operatorname{GOAL} Z$ \\
\hline 6.1 & $\mathrm{OF}_{\mathrm{A} 1}$ & $\mathrm{OF}_{\mathrm{A} 2}$ & $\mathrm{OF}_{\mathrm{A} 3}$ & $\mathrm{OF}_{131}$ & $\mathrm{OF}_{132}$ & & & & \\
\hline
\end{tabular}

determine the necessary duration (critical path) of a project. These directional linkages between activities are resource-bearing, and provide critical benchmarks which identify potential problems to project completion as each activity is operationalized. Examples of problems wherein precedent activity mapping has promoted utilitarian answers follow.

- Determine critical resource requirements of the critical path in order to define priority for resource availability.

- Determine non-critical resource requirements which might be redistributed to a critical path element, should that element's progress jeopardize the entire project map.

- Determine interrelationships between competing critical paths of multiple projects for strategic planning and global program (multiple projects) coordination and control.

\subsubsection{Technique}

Activities are defined by (a) observable actions required to satisfy the activity as stated, and (b) enumerated resources necessary to fulfill the requirements of each activity's actions. Each activity is designated by a unique numeric (or unique alpha) code, and those activities which immediately precede a subsequent activity identified using that code. This predecessorsuccessor link provides the basis for constructing an activity-on-node network, wherein resource-bearing nodes denote activities, and connective arcs (unidimensional arrows) denote precedence. Solution of the nodal network is trivial. A retrograde summation approar begins with the end (completion of project) node and proceeds backwards by summing $t$ time components of each node along any interconnected path through the network, a.ıd concluding with the start (beginning of project) node. Therefore, each possible sequence of interconnected nodes throughout the network defines a network path. The path with the largest time component sum -- that path which defines the anticipated completion time (duration) of the project, and therefore the path wherein any delay produces a delay in the anticipated completion of the project -- is defined as the critical path, and thus benchmarks the nodal network for monitoring the progress of the project towards completion. Simulation is achieved by reorganizing the nodal precedent linkages to study impact to the system; or, 
alternatively, by redistributing available resources in order to facilitate the completion of competing resource tasks. Decision support system status is achieved when the relationship between time-to-completion and resource expenditures satisfy the stated mission of the original project.

\subsubsection{Example}

A school district has decided, that a classroom building, mothballed some years ago during a period of severe declining enrollment, should be reconditioned for instructional use during the upcoming academic year because of projected demand due to escalating district enrollments. Due to the complex activities associated with reconditioning a building, and the extensive use of independent subcontracted companies, the district business manager has constructed an activity-on-node network to display graphically the full reconditioning project. Figure 4 illustrates a small part of this nodal network.

Figure 4. Activity-on-node network for instructional building reconditioning project.

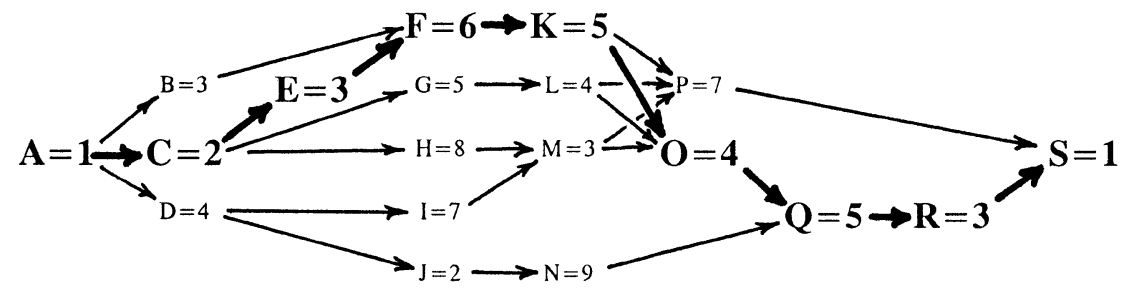

The critical path for this precedent activity mapping model is identified by the enlarged, bolded nodes along the critical path as shown in Figure 4, and as detailed in Figure 5.

The sum of the time components for each activity along this path equals 30 units, and by inspection can be proven to be the longest path through this network. Thus, the time necessary to complete this portion of the reconditioning project is 30 units. Other resources (personnel, equipment, materials, etc.) can be accumulated similar to the time component. An analysis of this network provides important information to the planner, in addition to identifying the critical path elements by activity nodal name. Slack for each activity, the amount of time an activity can be delayed without delaying the entire project's anticipated completion date, is calculated. In addition, actual calendar dates and 2400 clock times can be displayed for each of the elements inherent in slack calculations: earliest and latest start/finish times. Based on this benchmarking by initial critical path, any subsequent change to the network due to shift of resources, delay in activity start or completion, or related unanticipated problems, can be input to the model, and the model re-analyzed for updated benchmarking. 
Figure 5. Detailed listing of nodal elements and critical path.

\begin{tabular}{|c|c|c|c|c|c|c|c|}
\hline \multicolumn{2}{|c|}{ Activity } & Symb & Time & $\begin{array}{l}\text { Earliest } \\
\text { Start/Fin }\end{array}$ & $\begin{array}{l}\text { Latest } \\
\text { start/Fin }\end{array}$ & Slack & \multirow[b]{2}{*}{ critical } \\
\hline $\mathrm{ACT}$ & 1 & A & 1.0000 & $\begin{array}{l}0.0000 \\
1.0000\end{array}$ & $\begin{array}{l}0.0000 \\
1.0000\end{array}$ & 0.0000 & \\
\hline $\mathrm{ACT}$ & 2 & B & 3.0000 & $\begin{array}{l}1.0000 \\
4.0000\end{array}$ & $\begin{array}{l}3.0000 \\
6.0000\end{array}$ & 2.0000 & \\
\hline $\mathrm{ACT}$ & 3 & $\mathrm{C}$ & 2.0000 & $\begin{array}{l}1.0000 \\
3.0000\end{array}$ & $\begin{array}{l}1.0000 \\
3.0000\end{array}$ & 0.0000 & critical \\
\hline $\mathrm{ACT}$ & 4 & D & 4.0000 & $\begin{array}{l}1.0000 \\
5.0000\end{array}$ & $\begin{array}{l}3.0000 \\
7.0000\end{array}$ & 2.0000 & \\
\hline $\mathrm{ACT}$ & 5 & $\mathrm{E}$ & 3.0000 & $\begin{array}{l}3.0000 \\
6.0000\end{array}$ & $\begin{array}{l}3.0000 \\
6.0000\end{array}$ & 0.0000 & critical \\
\hline $\mathrm{ACT}$ & 6 & $F$ & 6.0000 & $\begin{array}{r}6.0000 \\
12.0000\end{array}$ & $\begin{array}{r}6.0000 \\
12.0000\end{array}$ & 0.0000 & critical \\
\hline $\mathrm{ACT}$ & 7 & G & 5.0000 & $\begin{array}{l}3.0000 \\
8.0000\end{array}$ & $\begin{array}{r}8.0000 \\
13.0000\end{array}$ & 5.0000 & \\
\hline & & & * REMA & DER OF LIST TE & MINATED * & & \\
\hline
\end{tabular}

\section{SUMMARY}

Mathematical decision support systems provide a wide variety of models which will (a) interface easily with data stored routinely by management information systems, (b) provide for simulating alternative solutions to a particular problem for review purposes, and (c) promote effective and efficient coordination and control in strategic planning in education. Linking a model with the existing management information system provides for continuous updating of the linked model, and therefore, continuous updated decisional options to the decision maker. As a result of these model interactions, the management information system can be supplied with new data from the analysis of the alternatives modeled. Perhaps the most significant benefit from the mathematical modeling of alternative decision situations within the context of a decision support system, is that of the decision maker's ability to simulate the impact of alternative solutions upon the problem environment. Simulating the probability of likely occurrences (effect) based upon alternative actions (cause) provides assurance that rational decision making is paralleled by critical thinking.

\section{REFERENCES}

Wholeben, B. E. (1993). Crisis prevention: Use operations research and be prepared. School Business Affairs, 59(9), 10-14.

Wholeben, B. E. (1990, March). Computer applications to educational planning and management. Invited address to the International Congress for Planning and Management of Educational Development, Mexico City, Mexico. Sponsored by the United Nations Educational, Scientific, and Cultural Organization (UNESCO).

Wholeben, B. E. (1985). Decision simulation modeling for educational administration (Report No. 105.458.4). Paris, France: United Nations Educational, Scientific, and Cultural Organization. 